Review began 02/10/2022 Review ended 02/14/2022 Published 02/21/2022

(๑) Copyright 2022

Jin et al. This is an open access article distributed under the terms of the Creative Commons Attribution License CC-BY 4.0., which permits unrestricted use, distribution, and reproduction in any medium, provided the original author and source are credited.

\section{A Single Axial Slice of the Sternocleidomastoids and Paravertebral Muscles Associated with Worse Local Progression-Free Survival and Severe Toxicity in Sarcopenic Head and Neck Cancer Patients Undergoing Radiotherapy}

William Jin ${ }^{1,2}$, Benjamin Rich ${ }^{1,2}$, Raphael Yechieli ${ }^{2}$, Laura Freedman ${ }^{2}$, Michael A. Samuels ${ }^{2}$, Matthew Abramowitz $^{2}$, Ruben Carmona ${ }^{2}$, Stuart E. Samuels ${ }^{2}$

1. Department of Radiation Oncology, Jackson Memorial Hospital, Miami, USA 2. Department of Radiation Oncology, University of Miami, Miami, USA

Corresponding author: William Jin, will.h.jin@gmail.com

\section{Abstract}

\section{Objective}

The objective of this study is to contrast the predictive ability of targeted muscle groups as radiographic proxies of sarcopenia on computerized tomography (CT) with body mass index (BMI) in head and neck cancer patients (H\&NCP) undergoing radiation at a safety net hospital, and to evaluate sarcopenia with survival, local progression, toxicities and treatment delays.

\section{Methods}

A retrospective review included $52 \mathrm{H} \& \mathrm{NCP}$ treated between 2017-2019. The posterior neck muscles (PN), sternocleidomastoids (SCM), and their summed volume (AM) were contoured at C3 on patients' pretreatment CT scans, then normalized to obtain skeletal muscle index (MI) values. Pre-treatment BMI was also evaluated. Cutoffs for sarcopenia were determined by receiver operating characteristic curves. Overall survival and local recurrence-free survival were evaluated by Kaplan-Meier. Acute grade 3 or higher toxicities were evaluated by binomial logistic regression.

\section{Results}

Using all neck muscles (AM-MI) produced the best model for predicting outcomes, outperforming individual muscle groups and BMI. Local progression-free survival was worse in sarcopenic patients at 25.81 months versus 35.40 months $(\mathrm{p}=0.026)$. Acute grade 3 or higher toxicities were associated with sarcopenia $(\mathrm{p}=0.005)$.

\section{Conclusions}

In this small, retrospective single-institution experience at a safety net hospital, a single axial slice of the combined sternocleidomastoids and paravertebral muscles at $\mathrm{C} 3$ performed better than either muscle group alone or pre-treatment BMI at predicting oncologic outcomes.

Categories: Otolaryngology, Radiation Oncology

Keywords: chemoradiation, radiotherapy, cachexia, head and neck cancer, sarcopenia

\section{Introduction}

Head and neck cancer patients (H\&NCP) must undergo one of the most difficult combined modality treatments for cancer. In a typical seven-week course of concurrent chemoradiation, up to 85\% [1] of patients develop symptoms severe enough to need active, supportive interventions. The process of cancer cachexia relies on pro-inflammatory cytokines like TNF- $\alpha$ [2], IL-1, and IL-6 to create a hypercatabolic state capable of meeting the anabolic demands of a perpetually growing tumor [3-5]. Without interventions, this leads to sarcopenia, a clinical phenotype characterized by a quantifiable loss of skeletal muscle mass and function [6]. Recent studies have shown the impact of sarcopenia on both treatment tolerance and outcomes in H\&NCP during chemoradiation [7-10]. The importance of this is understated, as sarcopenia is a potentially modifiable risk factor amenable to intervention.

Traditional metrics such as pre-treatment weight and body mass index (BMI) fail to reliably identify highrisk H\&NCP. Radiographic proxies for sarcopenia were first identified in gastrointestinal cancers using a single axial slice of the bilateral psoas muscles [11]. The large hip flexors were selected as proxies for sarcopenia for two reasons. These muscles were conveniently available as part of the standard diagnostic workup for gastrointestinal cancers. In addition, the hypothesis that form begets function was confirmed as 
the psoas correlated with both a quantitative loss of muscle mass and function. In gastrointestinal cancers, emerging evidence suggests correlations with both survival and toxicity [12-19]; however, an equivalent proxy for $\mathrm{H} \& \mathrm{NC}$ has yet to be determined.

The ideal muscle group should be reproducibly identified on cross-sectional imaging and functions in some capacity as a tonic (or postural) muscle. In contrast to phasic muscles, tonic muscles are critical to postural function and resistant to disuse atrophy, with size correlating to function. While there is growing evidence that the psoas muscles are applicable to H\&NCP undergoing chemoradiation $[9,20]$, most of these patients do not undergo high-resolution CT scans of the abdomen/pelvis as part of their oncologic workup. Efforts were directed to find a localized proxy in diagnostic H\&N scans [21,22]. A Pamukkale University study aimed to identify the ideal H\&N correlate to the psoas muscles. They looked at the paravertebral muscles (C2, C3, $\mathrm{C} 4$ ) and the sternocleidomastoids, revealing the $\mathrm{C} 3$ paravertebral muscles correlated best for men and sternocleidomastoids for women [23]. However, these previous studies used a semi-quantitative thresholding technique requiring images to be transferred to an external software program for analysis.

Our study aimed to identify a clinically meaningful cutoff for sarcopenia in our own institutional cohort of H\&NCP at a safety net hospital using clinically identified muscle groups and contrast the predictive value of the sternocleidomastoids (SCM) alone, the posterior neck (PN) muscles alone, and both with pre-treatment BMI. Finally, we aimed to evaluate the utility of the most optimal sarcopenia proxy with important endpoints, like treatment outcomes, tolerance, and delays. We hypothesized when identified, sarcopenia predicts worse local control, worse survival, increased acute toxicity, and increased delays in H\&NCP undergoing radiotherapy.

\section{Materials And Methods}

\section{Patient selection and treatment}

A retrospective review of an IRB-approved head and neck cancer database at a safety net hospital was performed. Sixty-three patients that underwent definitive head and neck treatment with radiation at a safety net hospital and with at least twelve months of follow-up were included. Then, nine patients were excluded because of secondary or metachronous cancers, prior history of cervical trauma, prior history of cervical radiotherapy, cervical kyphosis, cervical scoliosis, significant cervical positional lordosis, or paravertebral muscle invasion.

All patients were treated with radiation delivered via intensity-modulated radiation therapy. Use of chemotherapy was decided in a multidisciplinary tumor board. Demographic and outcomes data were obtained from the electronic medical record; radiation treatment data were collected from the Eclipse treatment planning system (TPS).

\section{Skeletal muscle delineation}

H\&N images used for radiation planning were transferred from the TPS to MiM version 6.7.6 64-bit edition (MIM Software Inc; Beachwood, OH). The first visible axial slice with the entire C3 vertebral body and the posterior process was chosen for contouring. Two muscle volumes were segmented: the PN and the bilateral SCM. A third volume (AM) was created from the sum of the PN and SCM. The PN included the longissimus capitis, levator scapulae, semispinalis capitis, and splenius capitus (Figure 1); transversospinales was excluded as it was visualized on some images entirely and trapezii were omitted as they are primarily phasic muscles. 


\section{Cureus}

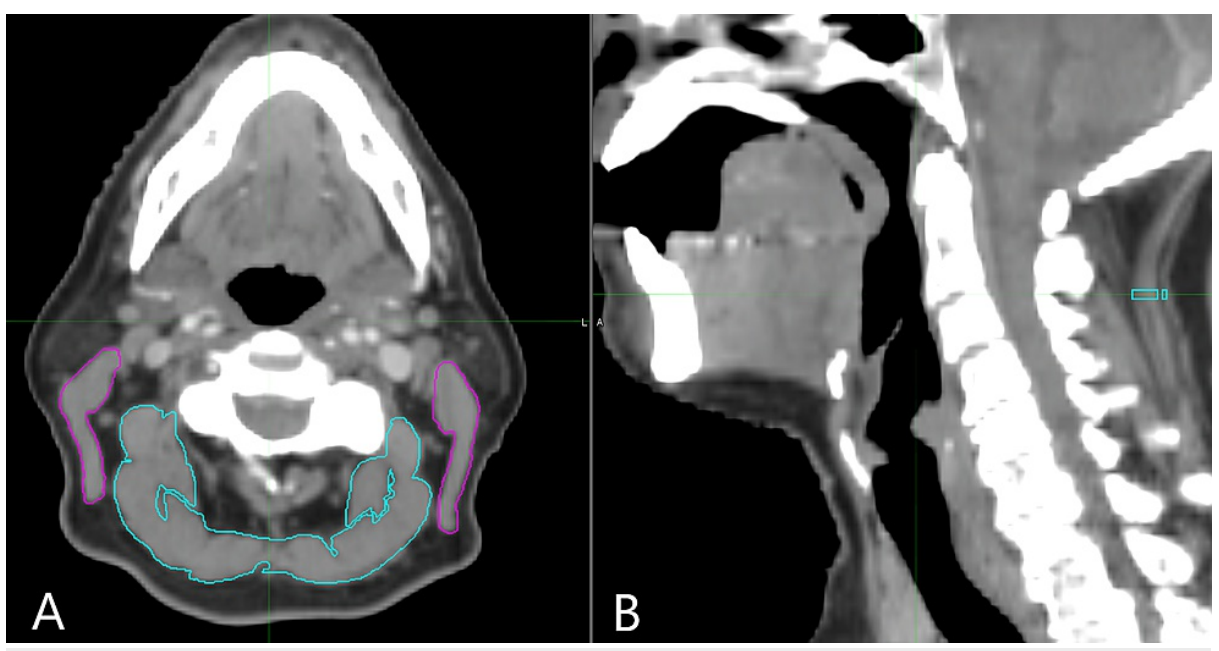

FIGURE 1: Delineation of muscle groups on axial and sagittal computerized tomography scans

Pre-treatment contrast-enhanced computer tomography scans with (A) axial view at the $\mathrm{C} 3$ vertebral body and (B) midplane sagittal view. Magenta highlights sternocleidomastoids and cyan highlights posterior neck muscles.

\section{Defining sarcopenia}

Muscle Indices (MI) were produced by normalizing by height using the equation shown below [12].

Muscle index $\left(\mathrm{mm}^{2} / \mathrm{m}^{2}\right)=$ muscle cross sectional area $\left(\mathrm{mm}^{2}\right) /$ patient height $\left(\mathrm{m}^{2}\right)$

Four indices were generated from running receiver operating characteristic (ROC) curves with respect to crude two-year local progression on each of the three segmented volumes (posterior neck muscle index (PN$\mathrm{MI}$ ), sternocleidomastoid muscle index (SCM-MI), sternocleidomastoid and posterior neck muscle combined muscle index (AM-MI)), as well as pre-treatment BMI. The index with the highest area under the curve (AUC) was selected for use in determining a binary categorical variable for sarcopenic status. The cutoff used was determined by the locus with the highest combination of sensitivity and specificity. Sarcopenia was defined as having a "low" muscle index.

\section{Outcomes}

Overall survival and local progression were calculated from the end of radiation treatment to a censoring event or most recent follow-up. Local progression events included persistence of disease or recurrence within the treatment field on clinical exam, nasopharyngoscopy, or imaging. Persistent weight loss was defined as significant weight loss (>10\% baseline) during treatment and failure to regain this weight six months after treatment. Toxicities were evaluated using Common Terminology Criteria for Adverse Events version 5. Toxicities occurring within three months of treatment were considered acute. Only severe toxicities, grade 3 and higher were included in this analysis. Treatment delays included any patient who missed two or more treatment days (excluding holidays and weekends).

All outcomes were stratified by possible confounders, including T stage, N stage, American Joint Committee on Cancer (AJCC) 8th edition staging, HIV status, gender, disease site, and presence of feeding tube prior to radiation.

\section{Statistical analysis}

Significance was set at $\mathrm{p}=0.05$. All statistics were performed on SPSS v23.0.0.2 64-bit edition (IBM Inc., Armonk, NY). Pearson Chi-square tests of independence were performed for patient and treatment characteristics, with respect to sarcopenic status. The Bonferroni method was applied when multiple variables were evaluated simultaneously. Cutoffs for continuous variables were performed with receiver operating characteristic curves. Time-to-event was evaluated using the Kaplan-Meier method. Toxicities were evaluated using binomial logistic regression.

\section{Results}

\section{Sarcopenic status}

The highest AUC of 0.790 belonged to AM-MI ( $p=0.005)$. The only other statistically significant index was 


\section{Cureus}

PN-MI (AUC=0.736, $\mathrm{p}=0.022)$. Both SCM-MI (AUC=0.679, $\mathrm{p}=0.082)$ and pre-treatment BMI (AUC=0.695, $\mathrm{p}=0.057$ ) performed worse than PN-MI (Figure 2). A cutoff of $9.3 \mathrm{~mm} 2 / \mathrm{m} 2$ was selected due to the highest combination of sensitivity (80.0\%) and specificity (69.0\%). Patients with a PN-MI lower than the cutoff were categorized as sarcopenic.

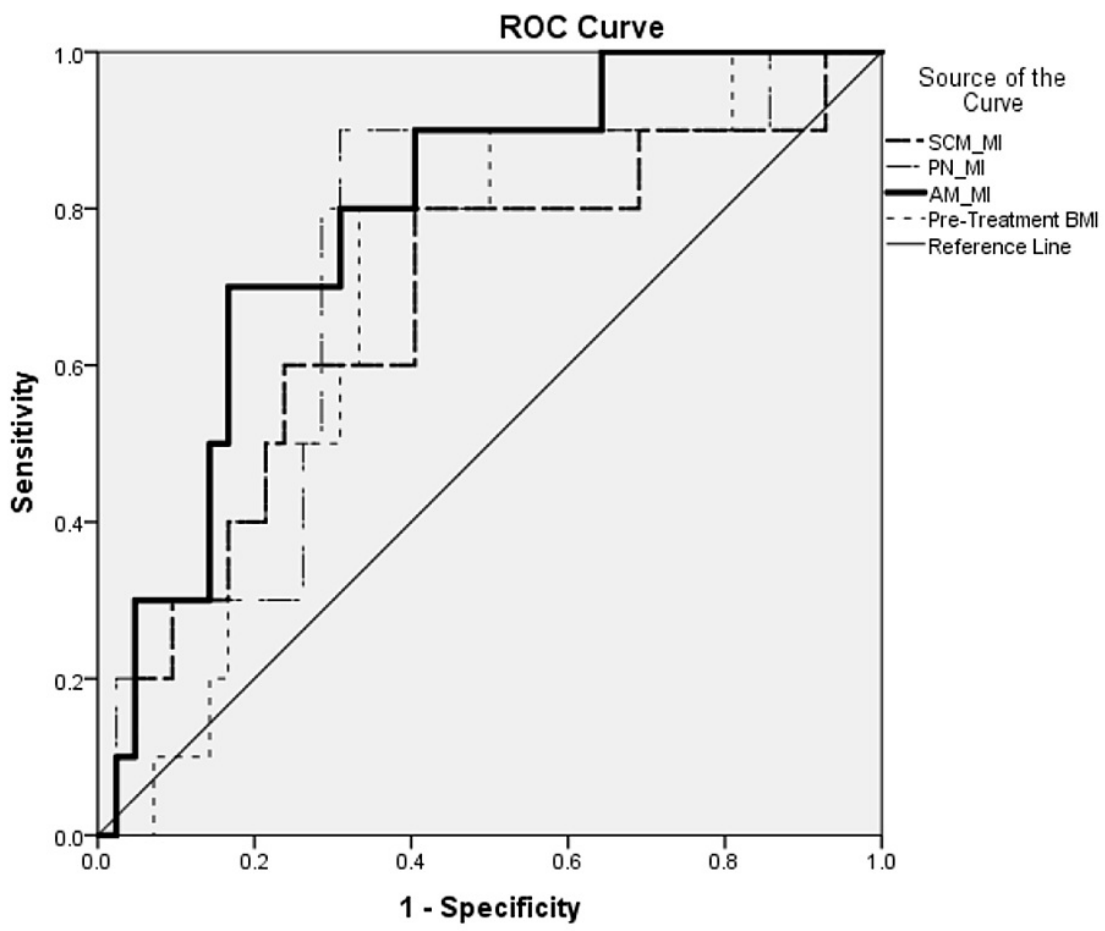

FIGURE 2: Receiver operating characteristic curve contrasting the sternocleidomastoids, the posterior neck muscles, all neck muscles combined, and pre-treatment body mass index

AM-MI: sternocleidomastoid and posterior neck muscle combined muscle index, BMI: body mass index, PN-MI: posterior neck muscle index, ROC: receiver operating characteristic, SCM-MI: sternocleidomastoid muscle index

\section{Patient and treatment characteristics}

As expected, sarcopenia correlated with lower BMI $(\mathrm{p}=0.007)$ and oral cavity location $(\mathrm{p}=0.006)$. However, no statistically significant differences were identified in other baseline characteristics, such as gender, HIV status, presence of feeding tube prior to radiation or TNM staging (Table 1). 


\section{Cureus}

\begin{tabular}{|c|c|c|c|c|}
\hline & & \multicolumn{2}{|c|}{ All muscle-muscle index } & \multirow{3}{*}{$p$-value } \\
\hline & & Sarcopenic & Non-sarcopenic & \\
\hline & & $\mathrm{n}(\%)$ & n (\%) & \\
\hline \multicolumn{2}{|l|}{ Age (median) } & 59 & 58 & NS \\
\hline \multirow{2}{*}{ Gender } & Male & $17(85)$ & $25(80.6)$ & \multirow{2}{*}{0.69} \\
\hline & Female & $3(15)$ & $6(19.4)$ & \\
\hline \multicolumn{2}{|l|}{ Pre-treatment BMI (median) } & 23.53 & 27.32 & 0.007 \\
\hline \multirow{4}{*}{ Anatomical site } & Oral cavity & $8(38.1)$ & $2(6.5)$ & \multirow{4}{*}{0.006} \\
\hline & Oropharynx & $4(19.0)$ & 18 (58.1) & \\
\hline & Hypopharynx/larynx & $8(38.1)$ & $11(35.5)$ & \\
\hline & Salivary gland & $1(4.8)$ & 0 & \\
\hline \multirow{4}{*}{ T stage } & T1 & $3(14.3)$ & $4(12.9)$ & \multirow{4}{*}{0.592} \\
\hline & $\mathrm{T} 2$ & $5(23.8)$ & 13 (41.9) & \\
\hline & T3 & $8(38.1)$ & $9(29.0)$ & \\
\hline & $\mathrm{T} 4$ & $5(23.8)$ & $5(16.1)$ & \\
\hline \multirow{4}{*}{$\mathrm{N}$ stage } & No & $5(23.8)$ & $9(29.0)$ & \multirow{4}{*}{0.592} \\
\hline & N1 & $4(19.0)$ & $7(22.6)$ & \\
\hline & N2 & $11(52.4)$ & $11(35.5)$ & \\
\hline & N3 & $1(4.8)$ & $4(12.9)$ & \\
\hline \multirow{4}{*}{ AJCC 8th staging } & Stage I & $1(4.8)$ & $4(12.9)$ & \multirow{4}{*}{0.219} \\
\hline & Stage II & $2(9.5)$ & $8(25.8)$ & \\
\hline & Stage III & $6(28.6)$ & $9(29.0)$ & \\
\hline & Stage IV-A/IV-B & $12(57.1)$ & $10(32.3)$ & \\
\hline \multirow{2}{*}{ PEG present before radiation } & No & $14(66.7)$ & $25(80.6)$ & \multirow{2}{*}{0.253} \\
\hline & Yes & $7(33.3)$ & $6(19.4)$ & \\
\hline \multirow{2}{*}{ HIV status } & HIV negative or unknown & $18(90.0)$ & $29(93.5)$ & \multirow{2}{*}{0.645} \\
\hline & HIV positive & $2(10.0)$ & $2(6.5)$ & \\
\hline
\end{tabular}

\section{TABLE 1: Baseline characteristics}

AJCC: American Joint Committee on Cancer, HIV: human immunodeficiency virus, PEG: percutaneous endoscopic gastrostomy, NS: not significant

Treatment characteristics were similar across both groups (Table 2). Concurrent chemoradiation was administered in $83.9 \%$ of our patients, with weekly cisplatin given in all but two cases, where carboplatin and paclitaxel were used in one and cetuximab used in the other. The median follow-up for the entire cohort was 30.22 months. 


\section{Cureus}

\begin{tabular}{|c|c|c|c|c|}
\hline & & \multicolumn{2}{|c|}{ All muscle-muscle index } & \multirow{3}{*}{ p-value } \\
\hline & & Sarcopenic & Non-sarcopenic & \\
\hline & & $\mathrm{n}(\%)$ & $\mathrm{n}(\%)$ & \\
\hline \multicolumn{2}{|l|}{ Radiation dose (Gy, median) } & 70 & 70 & NS \\
\hline \multicolumn{2}{|l|}{ Radiation fractions (median) } & 35 & 35 & NS \\
\hline \multicolumn{2}{|l|}{ Total elapsed radiation days (median) } & 47 & 49 & NS \\
\hline \multicolumn{2}{|l|}{ Days delayed (median) } & 2 & 2 & NS \\
\hline \multirow{2}{*}{ Concurrent chemo } & No & $3(15.8)$ & $4(12.9)$ & \multirow{2}{*}{0.775} \\
\hline & Yes & $16(84.2)$ & $27(87.1)$ & \\
\hline \multirow{2}{*}{ PEG tube placed during treatment } & No & $11(73.3)$ & $20(80.0)$ & \multirow{2}{*}{0.625} \\
\hline & Yes & $4(26.7)$ & $5(20.0)$ & \\
\hline \multirow{2}{*}{ Persistent weight loss } & No & $17(81.0)$ & 29 (93.5) & \multirow{2}{*}{0.163} \\
\hline & Yes & $4(19.0)$ & $2(6.5)$ & \\
\hline \multirow{2}{*}{ Post-op status } & No prior surgery & $10(47.6)$ & $22(71.0)$ & \multirow{2}{*}{0.089} \\
\hline & Post-op & $11(52.4)$ & $9(29.0)$ & \\
\hline
\end{tabular}

\section{TABLE 2: Treatment characteristics}

Chemo: chemotherapy, Gy: Gray, PEG: percutaneous endoscopic gastrostomy, post-op: post-operative, NS: not significant

\section{Outcomes}

Median local progression-free survival in sarcopenic patients was 25.81 months, while those without sarcopenia was 35.40 months ( $\mathrm{p}=0.026$, Figure 3). On univariate Cox regression, sarcopenia $(\mathrm{HR}=4.314$, $\mathrm{p}=0.003$ ) and percutaneous endoscopic gastrostomy (PEG) tube placed prior to treatment $(\mathrm{HR}=2.78, \mathrm{p}=0.039)$ were associated with local progression. On multivariate analysis, only sarcopenia was significant for local progression-free survival (LPFS) $(\mathrm{p}<0.001)$. 


\section{Cureus}

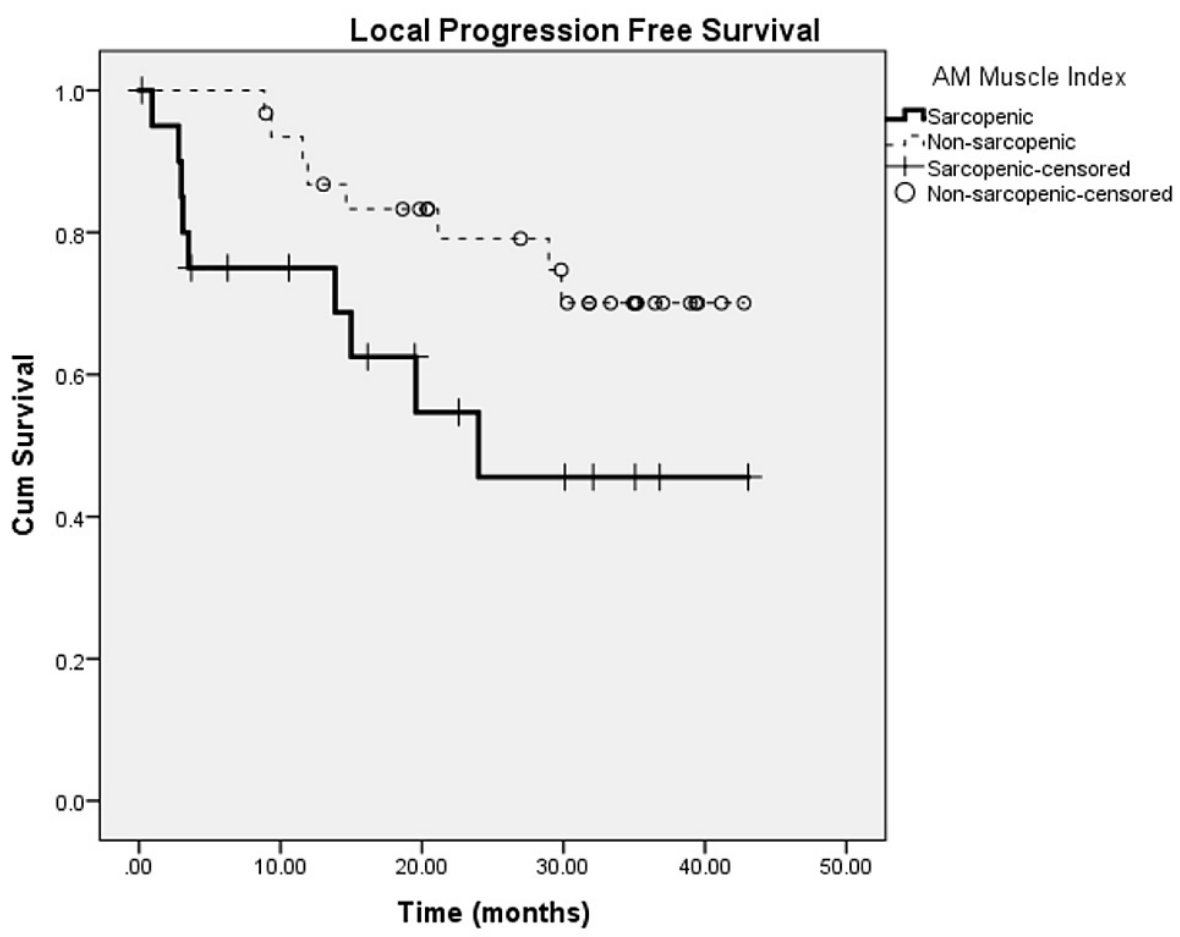

FIGURE 3: Kaplan-Meier curve for local progression-free survival between sarcopenic and non-sarcopenic patients

AM: sternocleidomastoid and posterior neck muscle combined

Median survival in sarcopenic patients was 34.41 months, while their counterparts with high AM-MI was 42.30 months ( $<<0.001$, Fig. 4). However, no censoring events occurred in the non-sarcopenic cohort (despite $\mathrm{n}=31$ ), and Cox regression was not performed. 


\section{Cureus}

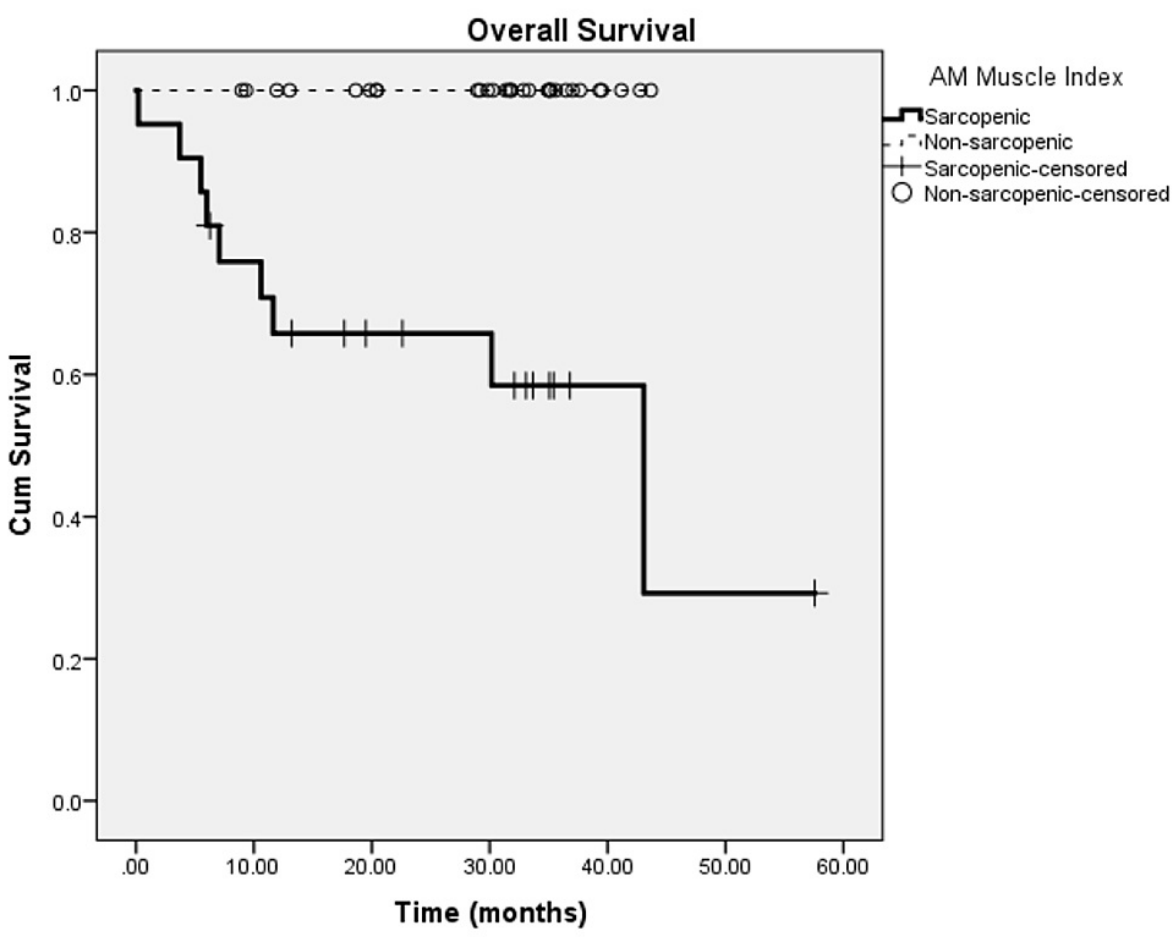

FIGURE 4: Kaplan-Meier curve for overall survival between sarcopenic and non-sarcopenic patients

AM: Sternocleidomastoid and posterior neck muscle combined

Acute grade $3+$ toxicities were significantly more common in the sarcopenic group (HR 5.71, $\mathrm{p}=0.008$ ). In the sarcopenic group, 10/21 (47.6\%) experienced grade 3 or higher toxicities, compared to 6/31 (19.4\%) patients in the non-sarcopenic group. In addition, persistent weight loss $(\mathrm{p}=0.181)$ or prolonged treatment delays $(\mathrm{p}=0.625)$ were not associated with sarcopenic patients.

\section{Discussion}

The psoas muscles' dominance as a proxy for sarcopenia/cachexia and predictive ability for outcomes in GI cancers led to a search for alternatives in H\&NCP with similar traits. The ideal candidate needed to be within the $\mathrm{H} \& \mathrm{~N}$ region, largely independent of patient positioning and resistant to disuse atrophy. The posterior neck muscles and sternocleidomastoids fit all requirements except for the centrally located transversospinales, which can vary significantly based on patient positioning. Ufuk et al. reported correlations between the sternocleidomastoids and paravertebral muscles with the psoas muscles [23]. Therefore, our study aimed to evaluate clinical correlates using the same muscle groups.

To our knowledge, this is the first study contrasting the clinically identified component muscle groups of the $\mathrm{H} \& \mathrm{~N}$ in relation to radiotherapy treatment outcomes in sarcopenic H\&NCP. Our results suggest that the combination of the SCMs and paravertebral muscles (AM-MI) identified sarcopenia more robustly than either muscle group alone or pre-treatment BMI. Moreover, all proxies of radiographic sarcopenia (SCM-MI, PN-MI, AM-MI) were better at predicting local progression. In our study, sarcopenic patients were more likely to locally progress and experience severe toxicities. For survival analysis, we saw a qualitative decrease in sarcopenic patients, but hesitate to draw statistical conclusions without a censoring event in the non-sarcopenic arm. Our initial working hypothesis was that sarcopenic patients are unable to tolerate treatment as well as non-sarcopenic patients. Then, increased rates of severe toxicities should lead to treatment delays, undertreated cancers from accelerated repopulation, higher rates of local progression, and ultimately, worse survival. However, both cohorts were similar in total treatment time; yet sarcopenic patients still progressed quicker. This suggests these patients either have a more biologically aggressive cancer, or there may be a metabolic threshold associated with successful radiotherapy treatment. Additional confirmatory studies are needed on both fronts.

Other contemporary retrospective studies used the sternocleidomastoids to predict for sarcopenic outcomes. Ganju et al. published the largest series to date with 246 H\&NCP [10]. Sarcopenia was associated with worse overall survival, progression-free survival, and radiation treatment breaks $>1$ week. Even in a high-risk sarcopenic population, Ganju et al. reported an unusually high (14\%) rate of radiation breaks of 
one week or longer. Our baseline and treatment characteristics were fairly like the University of Kansas study, with the only differences found in the proportion of obese patients and racial demographics. Patients in our study were primarily Hispanic, non-obese males compared to the University of Kansas' higher proportion of white, obese males. Sarcopenic obesity correlated with poor outcomes in the GI literature, but a Montefiore Medical Center published results detailing sarcopenic obesity as a protective factor for overall survival in H\&NCP [24]. Unfortunately, the literature does a poor job of defining sarcopenia, which may explain the heterogeneous results. Defining the volume of interest has been approached by using automated contouring of an entire axial slice, parts of an axial slice, or focused muscle groups, while cutoffs have been defined using population-based, cohort-based, disease site-based, and outcome-based values. Further studies with clear definitions for sarcopenia are needed to clarify their relationship to treatment outcomes.

The generalizability of our results is limited by its treatment in a safety net hospital (SNH), additional confounders, retrospective nature, and sample size. NCDB analyses found that patients treated at SNHs were associated with more advanced stages, poorer surgical outcomes, and racial minority demographics $[25,26]$. Confounders for sarcopenia secondary to cancer cachexia include geriatric sarcopenia [3], end-stage chronic obstructive pulmonary disease (COPD)-related cachexia, AIDS wasting syndrome, and poor mechanical intake, all of which are radiographically and clinically indistinguishable. Karsten et al. identified sarcopenia as a predictor for prolonged feeding tube dependency and is the only study thus far in H\&NCP [27]. Our results suggest that feeding tube insertion during radiation or before was not associated with sarcopenia. This may be due to variations with individual, institutional approaches towards multidisciplinary care. Forty-five percent of our sarcopenic patients were identified for high nutritional risk and underwent feeding tube placement; however, $75 \%$ of these patients had their feeding tubes placed even prior to ever setting foot in the radiation oncology department. Additionally, the highest risk patients for geriatric sarcopenia are over the age of 70 , few of which fell in our study $(n=6)$. For AIDS wasting syndrome, we are limited by the known HIV status of our patients since it is not a part of oncologic staging; however, out of the four patients with known HIV+, all were taking their anti-retroviral medications and had never experienced an AIDSdefining illness. Moreover, most patients received concurrent chemotherapy with cisplatin monitored by weekly complete blood counts (CBCs). In our patient population, 96\% were heavy smokers, with many comorbid for COPD. In summary, we acknowledge that confounders for cancer cachexia exist, but we are limited by the retrospective nature of our study to truly elucidate these differences. Despite our small sample size, the data generated were able to convey a strong signal with poor outcomes. All previously mentioned confounders were evaluated for effect modification via stratified Kaplan-Meier analysis, and no significant effect modifiers were found. Specifically for the oral cavity subsite, there was a correlation with sarcopenia but no effect modification on outcomes.

The literature suggests that sarcopenia secondary to cancer cachexia is highly prevalent in H\&NPC. Identifying a simple radiographic biomarker that can risk-stratify high-risk patients in need of interventional nutrition could improve oncologic outcomes. In addition to validation of these radiographic biomarkers in prospective studies, further studies should involve direct interventions to the cachectic process. Anti-inflammatory medications, anabolic/catabolic medications, nutritional/functional interventions are the four areas of ongoing research currently. An ongoing phase III trial in the UK called MENAC (Multimodal-Exercise, Nutrition and Anti-inflammatory medication for Cachexia) aims to utilize a holistic approach to an exceedingly complex problem [28].

\section{Conclusions}

In locally advanced H\&NCP undergoing radiotherapy at a safety net hospital, a single axial slice of the C3 paravertebral muscles or sternocleidomastoids can be used to identify sarcopenia. Sarcopenia was independently associated with worse local progression-free survival and severe toxicities. Additional prospective studies are needed to confirm the biology of tumors in sarcopenic patients, as well as the metabolic relationship to treatment outcomes.

\section{Additional Information Disclosures}

Human subjects: Consent was obtained or waived by all participants in this study. University of Miami Human Research Subjects Office issued approval 20200183. On 7/18/2020, the IRB reviewed and approved the following submission: Type of review: initial study. Title of study: Collection of Characteristics and Outcomes of Patients Treated for Head and Neck Cancer by Physicians at University of Miami and Jackson Memorial Hospitals. IRB ID: 20200183. Animal subjects: All authors have confirmed that this study did not involve animal subjects or tissue. Conflicts of interest: In compliance with the ICMJE uniform disclosure form, all authors declare the following: Payment/services info: All authors have declared that no financial support was received from any organization for the submitted work. Financial relationships: All authors have declared that they have no financial relationships at present or within the previous three years with any organizations that might have an interest in the submitted work. Other relationships: All authors have declared that there are no other relationships or activities that could appear to have influenced the submitted work. 


\section{References}

1. Adelstein DJ, Li Y, Adams GL, et al.: An intergroup phase III comparison of standard radiation therapy and two schedules of concurrent chemoradiotherapy in patients with unresectable squamous cell head and neck cancer. J Clin Oncol. 2003, 21:92-8. 10.1200/JCO.2003.01.008

2. Powrózek T, Mlak R, Brzozowska A, Mazurek M, Gołębiowski P, Małecka-Massalska T: Relationship between TNF- $\alpha-1031 \mathrm{~T} / \mathrm{C}$ gene polymorphism, plasma level of TNF- $\alpha$, and risk of cachexia in head and neck cancer patients. J Cancer Res Clin Oncol. 2018, 144:1423-34. 10.1007/s00432-018-2679-4

3. Argilés JM, Busquets S, Felipe A, López-Soriano FJ: Molecular mechanisms involved in muscle wasting in cancer and ageing: cachexia versus sarcopenia. Int J Biochem Cell Biol. 2005, 37:1084-104.

10.1016/j.biocel.2004.10.003

4. Couch ME, Dittus K, Toth MJ, et al.: Cancer cachexia update in head and neck cancer: pathophysiology and treatment. Head Neck. 2015, 37:1057-72. 10.1002/hed.23696

5. Peixoto da Silva S, Santos JM, Costa E Silva MP, Gil da Costa RM, Medeiros R: Cancer cachexia and its pathophysiology: links with sarcopenia, anorexia and asthenia. J Cachexia Sarcopenia Muscle. 2020, 11:61935. 10.1002/jcsm.12528

6. Meerkerk CD, Chargi N, de Jong PA, van den Bos F, de Bree R: Sarcopenia measured with handgrip strength and skeletal muscle mass to assess frailty in older patients with head and neck cancer. J Geriatr Oncol. 2021, 12:434-40. 10.1016/j.jgo.2020.10.002

7. Kwon M, Kim RB, Roh JL, et al.: Prevalence and clinical significance of cancer cachexia based on time from treatment in advanced-stage head and neck squamous cell carcinoma. Head Neck. 2017, 39:716-23. 10.1002/hed.24672

8. Orell-Kotikangas H, Österlund P, Mäkitie O, Saarilahti K, Ravasco P, Schwab U, Mäkitie AA: Cachexia at diagnosis is associated with poor survival in head and neck cancer patients. Acta Otolaryngol. 2017, 137:778-85. 10.1080/00016489.2016.1277263

9. Cho Y, Kim JW, Keum KC, Lee CG, Jeung HC, Lee IJ: Prognostic significance of sarcopenia with inflammation in patients with head and neck cancer who underwent definitive chemoradiotherapy. Front Oncol. 2018, 8:457. 10.3389/fonc.2018.00457

10. Ganju RG, Morse R, Hoover A, TenNapel M, Lominska CE: The impact of sarcopenia on tolerance of radiation and outcome in patients with head and neck cancer receiving chemoradiation. Radiother Oncol. 2019, 137:117-24. 10.1016/j.radonc.2019.04.023

11. Peng P, Hyder O, Firoozmand A, et al.: Impact of sarcopenia on outcomes following resection of pancreatic adenocarcinoma. J Gastrointest Surg. 2012, 16:1478-86. 10.1007/s11605-012-1923-5

12. Jin WH, Mellon EA, Frakes JM, et al.: Impact of sarcopenia in borderline resectable and locally advanced pancreatic cancer patients receiving stereotactic body radiation therapy. J Gastrointest Oncol. 2018, 9:24-34. 10.21037/jgo.2017.09.13

13. Murimwa GZ, Venkat PS, Jin W, et al.: Impact of sarcopenia on outcomes of locally advanced esophageal cancer patients treated with neoadjuvant chemoradiation followed by surgery. J Gastrointest Oncol. 2017, 8:808-15. 10.21037/jgo.2017.06.11

14. Nakanishi R, Oki E, Sasaki S, et al.: Sarcopenia is an independent predictor of complications after colorectal cancer surgery. Surg Today. 2018, 48:151-7. 10.1007/s00595-017-1564-0

15. Lanza E, Masetti C, Messana G, et al.: Sarcopenia as a predictor of survival in patients undergoing bland transarterial embolization for unresectable hepatocellular carcinoma. PLoS One. 2020, 15:e0232371. 10.1371/journal.pone.0232371

16. Barret M, Antoun S, Dalban C, et al.: Sarcopenia is linked to treatment toxicity in patients with metastatic colorectal cancer. Nutr Cancer. 2014, 66:583-9. 10.1080/01635581.2014.894103

17. Miyamoto Y, Baba Y, Sakamoto Y, et al.: Sarcopenia is a negative prognostic factor after curative resection of colorectal cancer. Ann Surg Oncol. 2015, 22:2663-8. 10.1245/s10434-014-4281-6

18. Prado CM, Baracos VE, McCargar LJ, et al.: Sarcopenia as a determinant of chemotherapy toxicity and time to tumor progression in metastatic breast cancer patients receiving capecitabine treatment. Clin Cancer Res. 2009, 15:2920-6. 10.1158/1078-0432.CCR-08-2242

19. Tan BH, Birdsell LA, Martin L, Baracos VE, Fearon KC: Sarcopenia in an overweight or obese patient is an adverse prognostic factor in pancreatic cancer. Clin Cancer Res. 2009, 15:6973-9. 10.1158/1078-0432.CCR09-1525

20. Findlay M, Brown C, De Abreu Lourenço R, White K, Bauer J: Sarcopenia and myosteatosis in patients undergoing curative radiotherapy for head and neck cancer: impact on survival, treatment completion, hospital admission and cost. J Hum Nutr Diet. 2020, 33:811-21. 10.1111/jhn.12788

21. Swartz JE, Pothen AJ, Wegner I, et al.: Feasibility of using head and neck CT imaging to assess skeletal muscle mass in head and neck cancer patients. Oral Oncol. 2016, 62:28-33. 10.1016/j.oraloncology.2016.09.006

22. Zwart AT, van der Hoorn A, van Ooijen PM, Steenbakkers RJ, de Bock GH, Halmos GB: CT-measured skeletal muscle mass used to assess frailty in patients with head and neck cancer. J Cachexia Sarcopenia Muscle. 2019, 10:1060-9. 10.1002/jcsm.12443

23. Ufuk F, Herek D, Yüksel D: Diagnosis of sarcopenia in head and beck computed tomography: cervical muscle mass as a strong indicator of sarcopenia. Clin Exp Otorhinolaryngol. 2019, 12:317-24. 10.21053/ceo.2018.01613

24. Fattouh M, Chang GY, Ow TJ, et al.: Association between pretreatment obesity, sarcopenia, and survival in patients with head and neck cancer. Head Neck. 2019, 41:707-14. 10.1002/hed.25420

25. Fangman BD, Goksu SY, Chowattukunnel N, et al.: Disparities in characteristics, access to care, and oncologic outcomes in young-onset colorectal cancer at a safety-net hospital. JCO Oncol Pract. 2021, 17:e614-22. 10.1200/OP.20.00777

26. Jassal JS, Cramer JD: Explaining racial disparities in surgically treated head and neck cancer . Laryngoscope. 2021, 131:1053-9. 10.1002/lary.29197

27. Karsten RT, Al-Mamgani A, Bril SI, et al.: Sarcopenia, a strong determinant for prolonged feeding tube 


\section{Cureus}

dependency after chemoradiotherapy for head and neck cancer. Head Neck. 2019, 41:4000-8.

10.1002/hed.25938

28. Solheim TS, Laird BJ, Balstad TR, et al.: Cancer cachexia: rationale for the MENAC (Multimodal-Exercise,

Nutrition and Anti-inflammatory medication for Cachexia) trial. BMJ Support Palliat Care. 2018, 8:258-65.

10.1136/bmjspcare-2017-001440 